\title{
Detecting reactivity
}

\author{
Michael G. Neubert, ${ }^{1,3}$ Hal Caswell, ${ }^{1}$ and Andrew R. Solow ${ }^{2}$ \\ ${ }^{1}$ Biology Department, Woods Hole Oceanographic Institution, Woods Hole, Massachusetts 02543-1049 USA \\ ${ }^{2}$ Marine Policy Center, Woods Hole Oceanographic Institution, Woods Hole, Massachusetts 02543 USA
}

\begin{abstract}
By definition, ecological systems at a stable equilibrium eventually return to the equilibrium point following a small perturbation. In the short term, however, perturbations can grow. Equilibria that exhibit transient growth following perturbation are said to be reactive. In this report, we present a statistical method for detecting reactivity from multivariate time series. The test is simple and computationally tractable, and it can be applied to short time series. Its main limitation is that it is based on a model of population dynamics that is linear on a logarithmic scale. Our results suggest that the test is robust when the dynamics are nonlinear on the log scale but that it may incorrectly classify an equilibrium as reactive when the reactivity is close to zero.
\end{abstract}

Key words: multivariate time series; resilience; stability; transient dynamics.

\section{INTRODUCTION}

If a biological system eventually returns to an equilibrium state after a small perturbation, the equilibrium is said to be stable. Stability of an equilibrium point, however, does not guarantee that small perturbations remain small. In fact, small perturbations to a stable equilibrium point may grow before they eventually decay. These transients can be large, driving the system far from its equilibrium state. If transient growth is possible, the equilibrium is said to be "reactive," and the maximum rate of growth of a perturbation is called the "reactivity" (Neubert and Caswell 1997).

The possibility of reactivity has practical consequences. For those charged with managing ecosystems, the knowledge that there may be dramatic short-term consequences of apparently small manipulations is obviously useful. Further, because ecological systems are more or less continuously buffeted by environmental perturbations, transient responses are likely to dominate our observations of real systems.

Reactivity is possible in both discrete-time and continuous-time systems. Reactivity has been studied in both discrete-time and continuous-time models of predator-prey and food-web dynamics (Chen and Cohen 2001, Neubert et al. 2004, Caswell and Neubert 2005, Verdy and Caswell 2008), spatial pattern formation (Neubert et al. 2002), aquatic ecology (Ives et al. 2003, Anderson et al. 2008), invasive species (Marvier et al. 2004), density-dependent matrix population models (Caswell and Neubert 2005), and epidemiology (Hosack et al. 2008). Caswell and Neubert (2005) and Verdy and

Manuscript received 4 November 2008; revised 27 March 2009; accepted 8 April 2009. Corresponding Editor: D. C. Speirs.

${ }^{3}$ E-mail: mneubert@whoi.edu
Caswell (2008) studied the sensitivity of reactivity to changes in model parameters.

Taken together, these studies indicate that reactivity is a common, but not universal, property of the equilibria of ecological and epidemiological models. In this report, we consider the statistical problem of estimating reactivity from time series data, and of testing the significance of the resulting estimate. To our knowledge, no formal statistical test for reactivity has been previously proposed. Our general approach is inspired by Solow and Sherman (1997) who developed a similar test for stability. We illustrate the test using three laboratory time series from the literature.

\section{REACTIVITY}

Let $\mathbf{x}_{t}$ be the vector of abundances of $k$ interacting populations or life history stages at discrete time $t$. A general deterministic model of the dynamics of this system is

$$
\mathbf{x}_{t}=f_{\boldsymbol{\theta}}\left(\mathbf{x}_{t-1}\right)
$$

where $f_{\boldsymbol{\theta}}$ is a nonlinear function with vector-valued parameter $\boldsymbol{\theta}$. A positive steady state $\boldsymbol{\mu}$ of the system in Eq. 1 is a solution to the following equation:

$$
f_{\boldsymbol{\theta}}(\boldsymbol{\mu})=\boldsymbol{\mu}
$$

with all elements of $\mu$ positive. We will ignore the case in which Eq. 2 has no positive solution.

If Eq. 1 is at steady state, then in the absence of an external perturbation, it will remain at steady state. Let $\mathbf{J}$ be the Jacobian matrix of partial derivatives of $f_{\boldsymbol{\theta}}$ evaluated at the steady state $\boldsymbol{\mu}$ :

$$
\mathbf{J}=\left(\frac{\partial f_{\boldsymbol{\theta}}}{\partial \mathbf{x}^{\prime}}\right)_{\mu} .
$$

In the neighborhood of $\boldsymbol{\mu}$, the dynamics of a perturbation $\mathbf{z}_{t}=\mathbf{x}_{t}-\boldsymbol{\mu}$ are approximated by the following: 


$$
\mathbf{z}_{t}=\mathbf{J z}_{t-1} .
$$

The steady state is locally stable if $\left\|\mathbf{z}_{t}\right\|$ vanishes in the limit of large $t$, which is guaranteed if all of the eigenvalues of $\mathbf{J}$ lie within the unit circle in the complex plane.

Caswell and Neubert (2005) defined the reactivity $v$ of the equilibrium $\boldsymbol{\mu}$ as the maximum rate of departure from $\boldsymbol{\mu}$ immediately following a perturbation, i.e.,

$$
v=\log \left(\max _{\left\|\mathbf{z}_{0}\right\| \neq 0} \frac{\left\|\mathbf{z}_{1}\right\|}{\left\|\mathbf{z}_{0}\right\|}\right)
$$

where $\|\cdot\|$ is the $l_{2}$ norm. They further showed that $v$ is easily computed as

$$
v=\log \sigma_{1}(\mathbf{J})
$$

where $\sigma_{1}(\mathbf{J})$ is the largest singular value of $\mathbf{J}$ (also called the spectral norm of $\mathbf{J}$ ). If $v>0$, the equilibrium point is reactive; if $v<0$ it is not.

The theory so far applies to time-invariant, deterministic systems. In order to develop a statistical method for detecting reactivity, we need to formulate a definition of reactivity for discrete-time stochastic systems. We begin with a standard stochastic version of the deterministic model in Eq. 1:

$$
\mathbf{X}_{t}=f_{\boldsymbol{\theta}}\left(\mathbf{X}_{t-1}\right) \exp \left(\boldsymbol{\varepsilon}_{t}\right)
$$

where $\boldsymbol{\varepsilon}_{t}$ is a $k$-variate normal process representing environmental noise. We assume $\boldsymbol{\varepsilon}_{t}$ has a mean vector $\mathbf{0}$ and variance matrix $\boldsymbol{\Sigma}$. (We will use uppercase symbols for random state variables and lowercase symbols for their realizations.) Let $\mathbf{Y}_{t}=\log \mathbf{X}_{t}$. Eq. 7 can be rewritten as follows:

$$
\mathbf{Y}_{t}=g_{\boldsymbol{\theta}}\left(\mathbf{Y}_{t-1}\right)+\boldsymbol{\varepsilon}_{t}
$$

where $g_{\boldsymbol{\theta}}(\mathbf{y})=\log f_{\boldsymbol{\theta}}[\exp (\mathbf{y})]$. We assume that Eq. 8 has a locally stable steady state $\boldsymbol{\mu}$. In analogy to the deterministic model (Eq. 1), $\boldsymbol{\mu}$ satisfies $g_{\boldsymbol{\theta}}(\boldsymbol{\mu})=\boldsymbol{\mu}$, and all of the eigenvalues of the Jacobian $\mathbf{A}=\left[\partial g_{\boldsymbol{\theta}} / \partial \mathbf{y}^{\prime}\right]$ evaluated at $\boldsymbol{\mu}$ lie within the unit circle in the complex plane. If the system (Eq. 8) is in the vicinity of $\boldsymbol{\mu}$, then it will remain in the vicinity of $\boldsymbol{\mu}$ provided the environmental noise is not too large. Moreover, in the vicinity of $\mu$,

$$
\mathbf{Y}_{t}=\boldsymbol{\mu}+\mathbf{A}\left(\mathbf{Y}_{t-1}-\boldsymbol{\mu}\right)+\boldsymbol{\varepsilon}_{t}
$$

to first approximation.

Eq. 9 is called a "first-order vector autoregressive [VAR(1)] model" (Reinsel 1997). It is the multivariate analogue of the familiar univariate first-order autoregressive [AR(1)] model. Ives et al. (2003) have made a forceful argument for basing statistical inference about stability properties (including reactivity) of multispecies communities on this model.

The reactivity of the stochastic model (Eq. 9) is also given by the logarithm of the largest singular value of $\mathbf{A}$. Suppose that $\mathbf{Y}_{t-1}$ is perturbed by the addition of a fixed vector $\boldsymbol{\delta}$. The magnitude of this vector is $\sqrt{\boldsymbol{\delta}^{\prime} \boldsymbol{\delta}}$. Replacing $\mathbf{Y}_{t-1}$ by $\mathbf{Y}_{t-1}+\boldsymbol{\delta}$ in Eq. 9 gives

$$
\mathbf{Y}_{t}=\boldsymbol{\mu}+\mathbf{A}\left(\mathbf{Y}_{t-1}-\boldsymbol{\mu}\right)+\mathbf{A} \boldsymbol{\delta}+\boldsymbol{\varepsilon}_{t} .
$$

The vector $\mathbf{A} \boldsymbol{\delta}$ represents the response of $\mathbf{Y}_{t}$ to the perturbation of $\mathbf{Y}_{t-1}$. The magnitude of this response is $\sqrt{\boldsymbol{\delta}^{\prime} \mathbf{A}^{\prime} \mathbf{A} \boldsymbol{\delta}}$. In analogy to Eq. 5, we can define the stochastic reactivity as the logarithm of the maximum of this amplification:

$$
\begin{aligned}
v & =\log \left(\max _{\|\boldsymbol{\delta}\| \neq 0} \sqrt{\frac{\boldsymbol{\delta}^{\prime} \mathbf{A}^{\prime} \mathbf{A} \boldsymbol{\delta}}{\boldsymbol{\delta}^{\prime} \boldsymbol{\delta}}}\right) \\
& =\log \sigma_{1}(\mathbf{A})
\end{aligned}
$$

where, as before, $\sigma_{1}(\mathbf{A})$ is the largest singular value of $\mathbf{A}$. Again, if $v>0$ then it is possible that a perturbation of the system in one period is amplified in the next and the system is called "reactive." If $v<0$, then no perturbation can grow and the system is called "nonreactive." Using a different argument, Ives et al. (2003) arrived at a similar definition of reactivity.

\section{A Test to Detect Reactivity}

Suppose that a set of observations are collected; these form an observed time series $\mathbf{y}_{t}$. The maximumlikelihood estimates of $\mathbf{A}, \boldsymbol{\mu}$, and $\boldsymbol{\Sigma}$, conditional on the initial observation $\mathbf{y}_{0}$, are given by standard results in time-series analysis (e.g., Anderson 1971: section 5.5.5):

$$
\hat{\mathbf{A}}=\left[\frac{1}{T} \sum_{t=1}^{T} \mathbf{y}_{t-1} \mathbf{y}_{t-1}^{\prime}-\overline{\mathbf{y}}_{(1)} \overline{\mathbf{y}}_{(1)}^{\prime}\right]^{-1}\left[\frac{1}{T} \sum_{t=1}^{T} \mathbf{y}_{t-1} \mathbf{y}_{t}^{\prime}-\overline{\mathbf{y}}_{(1)} \overline{\mathbf{y}}^{\prime}\right]
$$

$$
\begin{aligned}
& \hat{\boldsymbol{\mu}}=\left(\mathbf{I}_{k}-\hat{\mathbf{A}}\right)^{-1}\left[\overline{\mathbf{y}}-\hat{\mathbf{A}} \overline{\mathbf{y}}_{(1)}\right] \\
& \hat{\mathbf{\Sigma}}=\frac{1}{T} \sum_{t=1}^{T} \hat{\mathbf{w}}_{t} \hat{\mathbf{w}}_{t}^{\prime}
\end{aligned}
$$

where $\overline{\mathbf{y}}=(1 / T) \Sigma_{t=1}^{T} \mathbf{y}_{t}, \overline{\mathbf{y}}_{(1)}=(1 / T) \Sigma_{t=1}^{T} \mathbf{y}_{t-1}$, and $\hat{\mathbf{w}}_{t}=\mathbf{y}_{t}$ - $\hat{\boldsymbol{\mu}}-\hat{\mathbf{A}}\left(\mathbf{y}_{t-1}-\hat{\boldsymbol{\mu}}\right)$. It follows that the maximumlikelihood estimate of the reactivity is $\hat{v}=\log \sigma_{1}(\hat{A})$.

The value of $\hat{v}$ calculated from the observations gives the estimated reactivity. To assess the statistical significance of $\hat{v}$ we need the distribution of $v$ under a model corresponding, in some meaningful way, to the null hypothesis $H_{0}: v=0$. This distribution is obtained from an "adjusted" version of Eq. 9:

$$
\mathbf{Y}_{t}=\hat{\boldsymbol{\mu}}+\tilde{\mathbf{A}}\left(\mathbf{Y}_{t-1}-\hat{\boldsymbol{\mu}}\right)+\gamma_{t}
$$

where $\gamma_{t}$ is an independent sequence of $k$-variate normal random variables with mean 0 and variance $\hat{\boldsymbol{\Sigma}}$.

Eq. 13 has the same mean and the same covariance structure as the estimated process. However, the matrix $\tilde{\mathbf{A}}$ is chosen to have spectral norm $\sigma_{1}(\tilde{\mathbf{A}})=1$, and thus a reactivity of 0 . A test for reactivity rejects $H_{0}$ in favor of 
$H_{1}: v>0$ at significance level $\alpha$ if $\hat{v}$ lies above the upper $\alpha$ quantile of the sampling distribution of $v$ under Eq. 13.

There are a number of ways one might choose the adjusted matrix $\tilde{\mathbf{A}}$. A natural way would be to fit the model via maximum likelihood under the constraint $\sigma_{1}(\hat{\mathbf{A}})=1$. This, however, appears to be computationally intractable. An alternative approach is to choose $\tilde{\mathbf{A}}$ so that $\sigma_{1}(\tilde{\mathbf{A}})=1$, and $\tilde{\mathbf{A}}$ is as close as possible to $\hat{\mathbf{A}}$. We measure closeness with the Frobenius norm; i.e., we choose $\tilde{\mathbf{A}}$ so as to minimize

$$
\|\hat{\mathbf{A}}-\tilde{\mathbf{A}}\|_{F}=\left[\sum_{i=1}^{k} \sum_{j=1}^{k}\left(\hat{a}_{i j}-\tilde{a}_{i j}\right)^{2}\right]
$$

subject to the constraint $\sigma_{1}(\tilde{\mathbf{A}})=1$.

Here is how to construct such a matrix. The singular value decomposition of $\hat{\mathbf{A}}$ is

$$
\hat{\mathbf{A}}=\hat{\mathbf{U}} \hat{\mathbf{S}} \hat{\mathbf{V}}^{\prime} \text {. }
$$

If $\hat{\mathbf{A}}$ is nonsingular and has distinct singular values (which we assume) then $\hat{\mathbf{S}}$ is a diagonal matrix with positive entries $\sigma_{i}(\hat{\mathbf{A}})$, ordered so that $\sigma_{1}(\hat{\mathbf{A}})>\sigma_{2}(\hat{\mathbf{A}})>$ $\cdots>\sigma_{k}(\hat{\mathbf{A}})$. To create $\tilde{\mathbf{A}}$, we replace the matrix $\hat{\mathbf{S}}$ with $\tilde{\mathbf{S}}$, with diagonal elements $\sigma_{i}(\tilde{\mathbf{A}})$, such that

$$
\sigma_{i}(\tilde{\mathbf{A}})= \begin{cases}1 & \text { for } i=1, \\ \min \left\{1, \sigma_{i}(\hat{\mathbf{A}})\right\} & \text { for } i=2, \ldots, k\end{cases}
$$

The matrix

$$
\tilde{\mathbf{A}}=\hat{\mathbf{U}} \tilde{\mathbf{S}} \hat{\mathbf{V}}^{\prime}
$$

then has a largest singular value equal to one, and is as close to $\hat{\mathbf{A}}$ as possible (see Appendix A). It also has the desirable property of having the same singular vectors as $\hat{\mathbf{A}}$.

To generate a parametric bootstrap distribution of $v$ under the null hypothesis, we use Eq. 13 to generate a realization $\mathbf{y}_{1}^{*}, \mathbf{y}_{2}^{*}, \ldots, \mathbf{y}_{T}^{*}$. This simulated time series is used to obtain an estimate $\hat{\mathbf{A}}^{*}$ using Eq. $12 \mathrm{a}$, and the reactivity $\hat{\mathbf{V}}^{*}$ calculated as the logarithm of the largest singular value of $\hat{\mathbf{A}}^{*}$. This is repeated a large number of times (10000 in our example in Illustrations) and the observed value $\hat{v}$ is compared to the distribution of $\hat{v}^{*}$ under the null hypothesis.

\section{ILLUSTRATIONS}

We illustrate our test for reactivity by applying it to data from experiments on the flour beetle Tribolium as reported by Dennis et al. $(1997,2001)$. The beetles were grown in laboratory cultures and censused at two-week intervals. The data consists of a vector time series $\mathbf{y}_{t}$ with three components: the log-transformed numbers of larvae $\left(y_{1}\right)$, pupae $\left(y_{2}\right)$, and adults $\left(y_{3}\right)$. In Fig. $1 \mathrm{~A}, \mathrm{C}, \mathrm{E}$, we show the last 27 measurements of the log numbers in each life history stage for three control replicates (Cushing et al. 2003: Table A.18). For these replicates, the asymptotic dynamics were judged to be a stable equilibrium. We excluded the first 14 measurements from these series as they were far from the estimated equilibrium point. We fit the VAR(1) model (Eq. 9) to these data using Eq. 12a-c. The estimates of the parameters are presented in Appendix B. In Fig. $1 \mathrm{~B}, \mathrm{D}, \mathrm{F}$, we show the estimated reactivity $\hat{v}$ and the sampling distribution of $\hat{v}$ under the null hypothesis obtained from 10000 bootstrap replicates for each series.

The estimates of reactivity for the three beetle time series are all positive and remarkably similar. For replicate 4 (Fig. 1A, B), 19\% of the bootstrap estimates under the null hypothesis exceed the maximum-likelihood estimate $(\hat{v}=2.03)$ and we would not reject $H_{0}$. For replicates 11 and 24 (taken separately), however, evidence for a reactive equilibrium is much stronger. The estimates $\hat{v}$ (2.37 and 1.96, respectively) fall in the top 5\% and $2 \%$ of the bootstrap estimates under the null hypothesis.

\section{NONLINEARITY}

The simplicity of the test we have described here relies on the linearity of the VAR(1) model (Eq. 9), which is appropriate if the observations are close to equilibrium. On the other hand, real data are likely to contain some effects of nonlinearity, which is a common feature of ecological models. To assess the potential effects of nonlinearity on the performance of our test, we performed a simulation experiment using a discrete-time competition model. Let $x_{1}, x_{2}$ and $x_{3}$ be densities of three competing species. Then

$$
\begin{aligned}
& x_{1}(t)=x_{1} \exp \left[r\left(1-x_{1}-\alpha_{1} x_{2}-\beta_{1} x_{3}\right)+\varepsilon_{1}(t)\right] \\
& x_{2}(t)=x_{2} \exp \left[r\left(1-\beta_{2} x_{1}-x_{2}-\alpha_{2} x_{3}\right)+\varepsilon_{2}(t)\right] \\
& x_{3}(t)=x_{3} \exp \left[r\left(1-\alpha_{3} x_{1}-\beta_{3} x_{2}-x_{3}\right)+\varepsilon_{3}(t)\right]
\end{aligned}
$$

where the $x_{i}$ on the right-hand sides are evaluated at time $t-1$, and the environmental noise variables $\varepsilon_{i}(t)$ are independent normal random variables with zero mean and common variance $\sigma^{2}$. This model is a stochastic, dimensionless version of a model formulated by May and Leonard (1975). The deterministic version of the model has a unique positive equilibrium point $\mathbf{x}^{*}$. Roeger (2005) has derived conditions on the parameters that guarantee stability of the fixed point.

We want to compare the performance of the test when applied to data generated from the nonlinear model (Eq. 18a) and its linearization. To do so, we chose parameter sets $\left(r \in(0,2), \alpha_{i} \in(0,2), \beta_{i} \in(0,2)\right)$ that produced a range of reactivities. For each set of parameters, we set $\mathbf{x}_{0}=\mathbf{x}^{*}$ and generated 500 vector time series from the nonlinear model (Eq. 18) and an additional 500 time series from the linear VAR(1) model obtained by linearizing Eq. 18 around its equilibrium point. For each series we tested for reactivity at the 0.1 significance level using 500 bootstrap replicates. We recorded the fraction of the 500 simulations for which the null

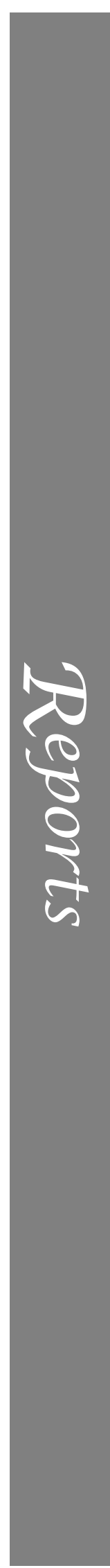



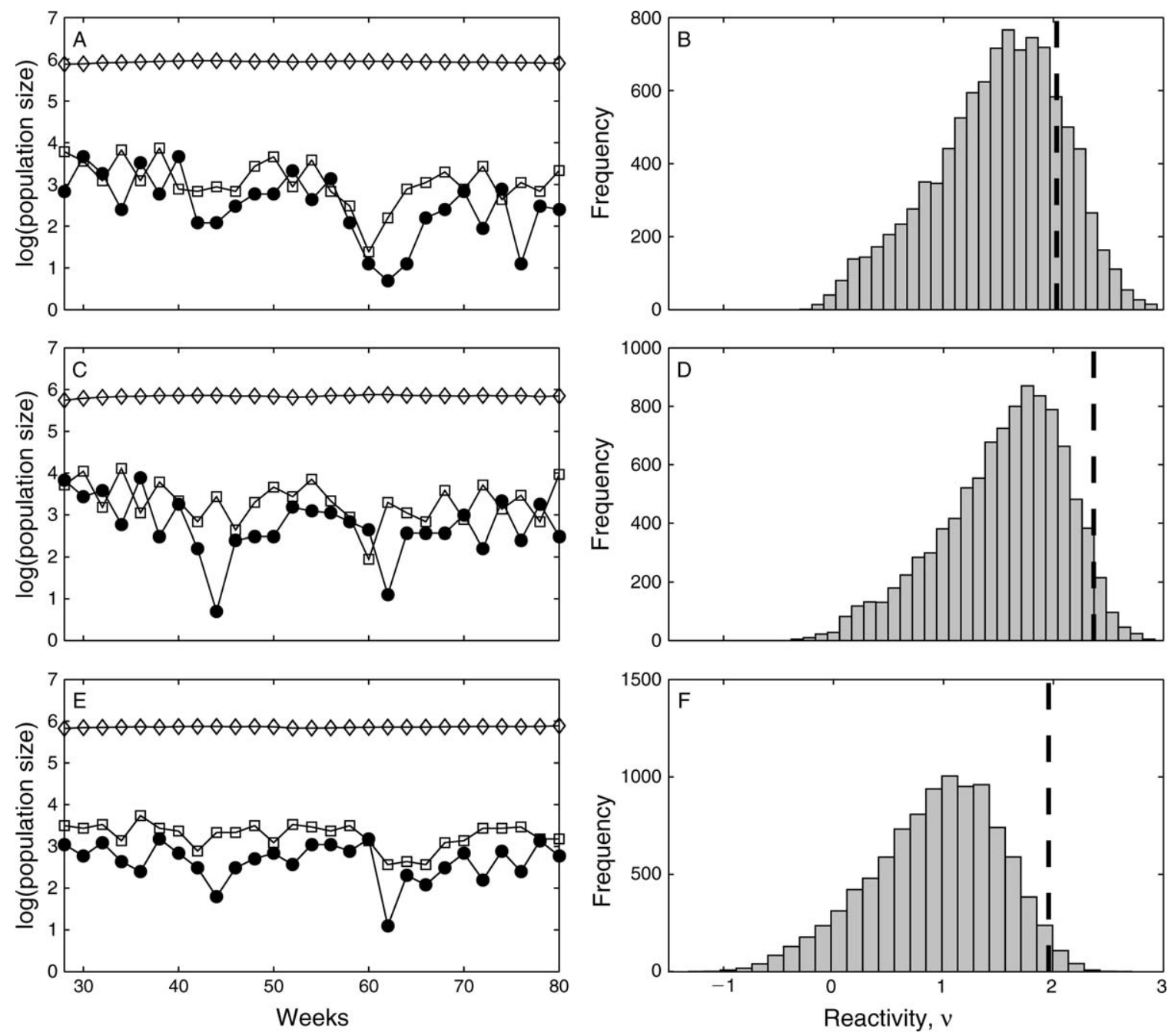

FIG. 1. (A, C, E) Time series of larval (open squares), pupal (solid circles), and adult (open diamonds) flour beetles from the experiments of Dennis et al. (1997) and reported in Cushing et al. (2003). (B, D, F) Estimates of the reactivity ( $v$, dashed line) and the sampling distributions of the reactivity under the null hypothesis $(v=0)$. Each row $(\mathrm{A}$ and $\mathrm{B}, \mathrm{C}$ and $\mathrm{D}, \mathrm{E}$ and $\mathrm{F})$ represents one control replicate.

hypothesis $\left(H_{0}: v=0\right)$ was rejected. The differences between the outcomes of these simulation experiments reveal the effects of nonlinearity. Performance of the test will be affected by the variance $\sigma^{2}$ and the length of the time series, so we examined cases with low $(\sigma=0.01)$ and high $(\sigma=0.1)$ variance and with short $(T=25)$ and long $(T=100)$ series. The results are summarized in Fig. 2.

The test performs well, at least for this model. For longer time series $(T=100$, Fig. 2A, C), the null hypothesis was never incorrectly rejected when reactivity was moderately low $(<-0.5)$ and was always correctly rejected when reactivity was moderately large $(>0.5)$. Nonlinearity did not seem to have an effect for these series. For reactivities in the neighborhood of zero, however, nonlinearity did have an important effect. The effect was particularly strong when reactivity was negative. In these cases, the test applied to the nonlinear model incorrectly rejected the null hypothesis at higher rates than when it was applied to the linear model. For shorter time series ( $T=25$, Fig. 2B, D), the effects of nonlinearity were apparent for positive as well as for moderately negative reactivities. A decrease in the length of the time series tended to diminish the ability of the test to detect a reactive equilibrium when it was in fact reactive.

\section{DisCUSSION}

Reactivity is an important aspect of the transient behavior of ecological systems, a class of behavior that is of increasing interest in ecology. Theoretical studies have shown that reactivity is a common property of models of populations, communities, and ecosystems (Neubert and Caswell 2000, Chen and Cohen 2001, Neubert et al. 2004, Caswell and Neubert 2005, Verdy and Caswell 2008). In this note we have described what 

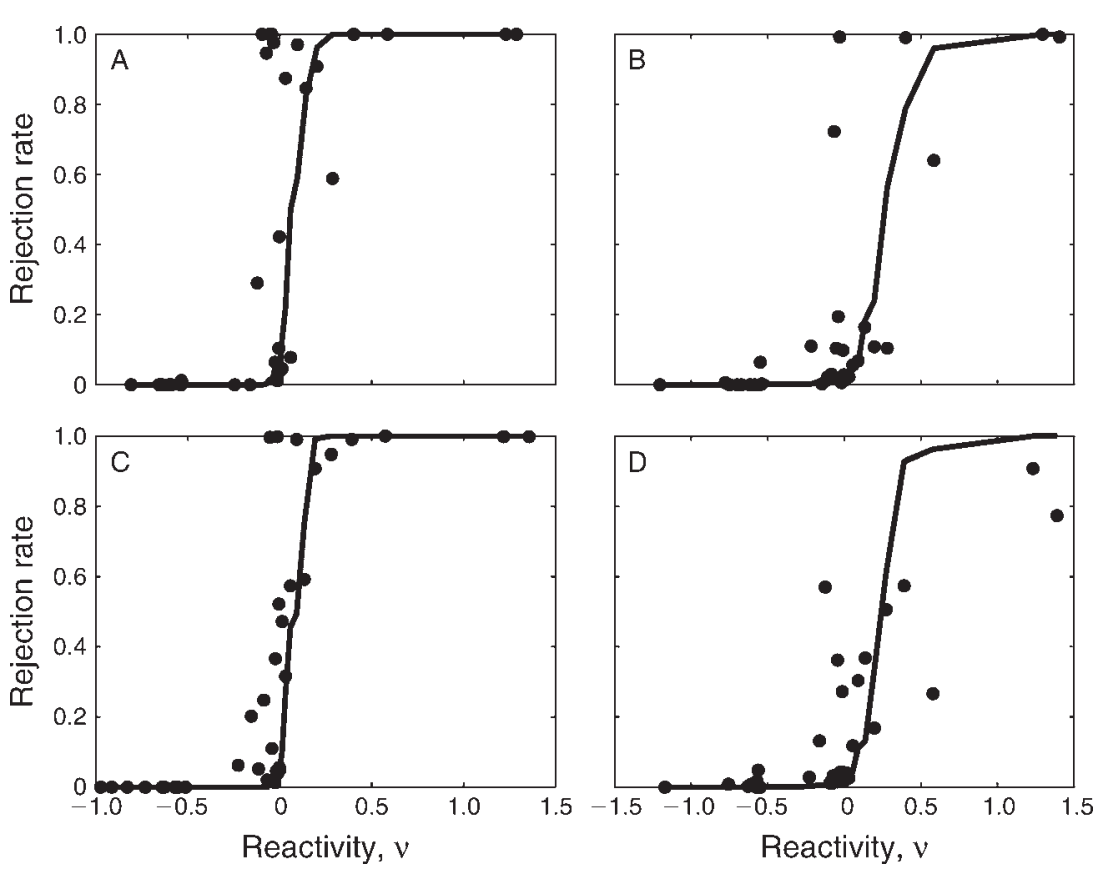

FIG. 2. Performance of the reactivity test as applied to data simulated from the nonlinear discrete-time May-Leonard competition model (Eq. 18) (solid circles) and its linearization (curve). The rate of rejection of the null hypothesis of no reactivity $\left(H_{0}: v=0\right)$ is plotted as a function of the actual reactivity $(v)$. Parameter values are: (A) $T=100, \sigma=0.01 ;(\mathrm{B}) T=25, \sigma=0.01 ;(\mathrm{C})$ $T=100, \sigma=0.1$; (D) $T=25, \sigma=0.1$; where $\sigma$ is the variance of the environmental noise and $T$ is the length of the time series. Other parameters were chosen as described in Nonlinearity.

is, to our knowledge, the first formal statistical test for reactivity.

Conceptually, there exist two complementary approaches to estimating reactivity. One is to develop a full nonlinear dynamic model of the system and estimate its parameters. Given the model, one solves for the equilibrium, evaluates the linear approximation there, and calculates reactivity from the Jacobian matrix. This can be applied to either experimental or field data. It does not require that the system be in the neighborhood of an equilibrium, indeed, it requires observations over a wide range of values of the state variables.

The second approach is to estimate the linear approximation directly, using the vector autoregressive time series approach we use here. This has the advantage of providing a statistical test of the null hypothesis that reactivity is zero. As such, the results we have presented here are in the same category as statistical time-series tests for density-dependence (Bulmer 1975) and stability (Solow and Sherman 1997). Our model was inspired by the Solow-Sherman test, and like that test, ours is simple and computationally tractable, and can be applied to short time series. Our test's main limitation is that it is based on a model of population dynamics that is linear on a logarithmic scale. This is most accurate when the data consist of observations in the vicinity of equilibrium. Our results suggest that the test is robust when the dynamics are nonlinear on the log scale, but that it may incorrectly classify an equilibrium as reactive when the reactivity is close to zero.

\section{ACKNOWLEDGMENTS}

This research was supported by a grant (DEB-0515639) from the U.S. National Science Foundation. The authors acknowledge Nicholas Higham for helpful suggestions regarding the matrix adjustment.

\section{Literature Cited}

Anderson, T. W. 1971. The statistical analysis of time series. John Wiley and Sons, New York, New York, USA.

Anderson, K. E., R. M. Nisbet, and E. McCauley. 2008. Transient responses to spatial perturbations in advective systems. Bulletin of Mathematical Biology 70:1480-1502.

Bulmer, M. G. 1975. The statistical analysis of density dependence. Biometrics 31:901-911.

Caswell, H., and M. G. Neubert. 2005. Reactivity and transient dynamics of discrete-time ecological systems. Journal of Difference Equations and Applications 11:295-310.

Chen, X., and J. E. Cohen. 2001. Transient dynamics and foodweb complexity in the Lotka-Volterra cascade model. Proceedings of the Royal Society B 268:869-877.

Cushing, J. M., R. F. Costantino, B. Dennis, R. A. Desharnais, and S. M. Henson. 2003. Chaos in ecology: experimental nonlinear dynamics. Academic Press, San Diego, California, USA.

Dennis, B., R. A. Desharnais, J. M. Cushing, and R. F. Costantino. 1997. Transitions in population dynamics: equilibria to periodic cycles to aperiodic cycles. Journal of Animal Ecology 66:704-729.

Dennis, B., R. A. Desharnais, J. M. Cushing, S. M. Henson, and R. F. Costantino. 2001. Estimating chaos and complex dynamics in an insect population. Ecological Monographs 71:277-303.

Hosack, G. R., P. A. Rossignol, and P. van den Driessche. 2008. The control of vector-borne disease epidemics. Journal of Theoretical Biology 255:16-25. 
Ives, A. R., B. Dennis, K. L. Cottingham, and S. R. Carpenter. 2003. Estimating community stability and ecological interactions from time-series data. Ecological Monographs 73: 301-330.

Marvier, M., P. Kareiva, and M. G. Neubert. 2004. Habitat destruction, fragmentation, and disturbance promote invasion by habitat generalists in a multispecies metapopulation. Risk Analysis 24:869-878.

May, R. M., and W. J. Leonard. 1975. Nonlinear aspects of competition between three species. SIAM Journal of Applied Mathematics 29:243-253.

Neubert, M. G., and H. Caswell. 1997. Alternatives to resilience for measuring the responses of ecological systems to perturbations. Ecology 78:653-665.
Neubert, M. G., T. Klanjscek, and H. Caswell. 2004. Reactivity and transient dynamics of predator-prey and food web models. Ecological Modelling 179:29-38.

Neubert, M. G., J. D. Murray, and H. Caswell. 2002. Transient dynamics and pattern formation: reactivity is necessary for Turing instabilities. Mathematical Biosciences 175:1-11.

Reinsel, G. 1997. Elements of multivariate time series analysis. Springer, New York, New York, USA.

Roeger, L.-I. W. 2005. Discrete May-Leonard competition models II. Discrete and Continuous Dynamical SystemsSeries B 5:841-860.

Solow, A. R., and K. Sherman. 1997. Testing for stability in a predator-prey system. Ecology 78:2624-2627.

Verdy, A., and H. Caswell. 2008. Sensitivity analysis of reactive ecological dynamics. Bulletin of Mathematical Biology 70: $1634-1659$.

\section{APPENDIX A}

Derivation of Eqs. 16 and 17 (Ecological Archives E090-189-A1).

\section{APPENDIX B}

Maximum-likelihood estimates for the time series of Fig. 1 (Ecological Archives E090-189-A2). 